\section{One and the same}

Proc. Natl Acad. Sci. USA http://doi.org/cs5k (2018)

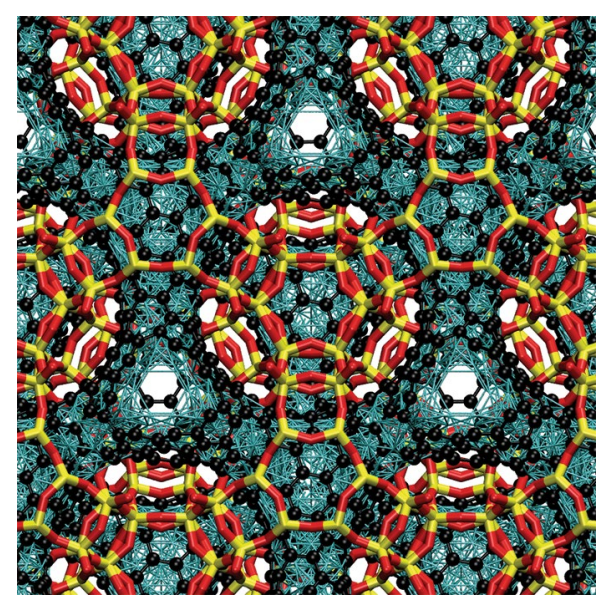

Credit: PNAS

Nanostructured carbon comes in many forms with different curvatures, such as positively curved spherical fullerenes or zero-curved two-dimensional graphene. Many theoretically predicted carbons adopt the form of so-called schwarzites, carbon allotropes with negative Gaussian curvature. However, it remains unclear whether 'random schwarzites' and zeolite-templated carbons (ZTCs), the only experimentally isolated materials with similar properties, can be considered schwarzites. Now, E. Braun and colleagues have presented a theoretical framework offering a complete library of synthesizable ZTCs from known zeolites, and confirmed that ZTCs are schwarzites incarnate.
The researchers exploit the idea of structural similarity between ZTCs and schwarzites, which resemble a triply periodic minimal surface (TPMS). The designed Monte Carlo method closely mimics the zeolite templating process. As an output, a subset of novel ZTCs was obtained and matched with the experimentally isolated ZTCs. Consequently, the researchers concluded that many ZTCs, despite their dissimilar structural parameters, resembled known TPMSs linking these materials to hypothesized carbon schwarzites and showing how to find template zeolites to experimentally synthesize a desired schwarzite.

https://doi.org/10.1038/s41565-018-0264-2

\section{ATOMIC IMAGING \\ Seeing through the noise \\ Phys. Rev. Lett. 121, 056101 (2018)}

Scanning transmission electron microscopy (STEM) with aberration correction can map atomic order with sub-ångström resolution. Yet, many nanomaterials are sensitive to high-energy electrons, which limits the applicable electron dose. This results in images that present weak contrast, hindering an unambiguous determination of the atomic structure. Jarmo Fatermans et al. now propose an advanced fitting procedure that enables a more reliable and unbiased detection of single atoms in high-noise, lowcontrast electron microscopy images.

The researchers combine a known model fitting with an advanced statistical selection method and apply this to STEM images of

\title{
FLUORESCENCE SPECTROSCOPY
}

\section{A single object rotating}

Nano Lett. 18, 5279-5287 (2018)

Single molecules can be trapped inside a microfluidic chamber by an electrokinetic potential that counteracts the Brownian motion. A fluorescence signal determines the position of the particle, and by measuring the feedback forces researchers can determine the translational diffusion coefficient, which is related to the hydrodynamic radius of the trapped particle. However, a parameter that is more sensitive to the size of an object is the rotational diffusivity. Yang and Moerner have now shown how to measure the rotational diffusivity of a trapped object, and evaluate its volume.

The researchers use an electrokinetic trap, but they modify it to detect the timeresolved fluorescent signal from two polarization channels. As the molecule freely rotates, the two polarized signals will decay over time with kinetics that depend, among other parameters, on the rotational correlation time. By fitting the decay curves, the researchers can determine the rotational diffusivity and hence the volume of the object, and can recognize single- from double-stranded DNA from a mixture of the two with $80 \%$ accuracy.

https://doi.org/10.1038/s41565-018-0265-1

atomic-scale systems. The fitting approximates the contrast of atomic columns in an STEM image with Gaussian functions on a constant background. Then, they introduce an approximate analytical implementation of a probability rule, which, based on the pixel intensities, determines the probability for a specific number of atomic columns to give rise to this image. The result is a set of configurations with different numbers of atomic rows and their position together with the probability for each configuration. This method is more reliable than conventional peak-fitting methods, avoids the bias in visual inspection and provides the likeliness of one atomic configuration compared to alternative structures.

https://oi.org/10.1038/s41565-018-0263-3

\section{SYNTHETIC BIOLOGY PLATFORM Boost microbiota studies Nat. Commun. 9, 3347 (2018)}

The gut microbiota has attracted broad attention due to its high relevance to human health and disease. Next-generation deep sequencing in combination with bioinformatics is widely used to study the working mechanism of the microbiome. However, these techniques are costly, slow and complex. Collins and co-workers report the extended usage of their paper-based synthetic biology platform, which is much cheaper, more on-demand and simpler, for analysing microbial and host RNAs in complex biological samples.

This sensing platform includes two key steps: nucleic acid sequence-based amplification and RNA toehold switch-mediated sensing. The researchers find that identifying bacterial species via the detection of $16 \mathrm{~S}$ ribosomal RNAs does not work here due to the significant crosstalk that occurs among related bacterial species, which can be attributed to similarities in their trigger RNA sequences. To address this specificity issue, they perform bioinformatics analysis and identify the species-unique mRNAs of ten bacteria that are relevant to microbiota studies. By normalizing to a single standard, they can measure the target RNAs semi-quantitatively via the above-mentioned two steps. This is validated on stool samples from inflammatory bowel disease patients, with the determined microbial and host mRNA concentrations well correlated with reverse transcription-qPCR. 